\title{
Modelling of the hepatic circulation by combining vascular corrosion casting and micro-CT imaging
}

\section{Charlotte Debbaut ${ }^{1}$, Christophe Casteleyn ${ }^{2}$, Pieter Cornillie ${ }^{3}$, Denis Van Loo ${ }^{4,5}$, Jan Vierendeels ${ }^{6}$, Patrick Segers ${ }^{1}$, and Diethard Monbaliu ${ }^{7}$}

${ }^{1}$ Biofluid, Tissue and Solid Mechanics for Medical Applications (bioMMeda), Institute Biomedical Technology, Ghent University. De Pintelaan 185 - Block B, B-9000 Gent, Belgium Email: charlotte.debbaut@ugent.be; telephone: (0032) 93323379

${ }^{2}$ Laboratory for Veterinary Anatomy, University of Antwerp. Universiteitsplein 1, 2610 Wilrijk, Belgium

${ }^{3}$ Applied Veterinary Morphology, Faculty of Veterinary Medicine, Ghent University. Salisburylaan 133, B-9820 Merelbeke, Belgium

${ }^{4}$ Centre for X-Ray Tomography, Department of Physics and Astronomy, Ghent University. Proeftuinstraat 86, B-9000 Gent, Belgium

${ }^{5}$ Department of Soil Management, Ghent University. Coupure links 653, B-9000 Gent, Belgium

${ }^{6}$ Department of Flow, Heat and Combustion Mechanics, Ghent University. SintPietersnieuwstraat 41, B-9000, Gent, Belgium

${ }^{7}$ Department of Abdominal Transplant Surgery, University Hospitals Leuven, Catholic University Leuven. Herestraat 49, B-3000 Leuven, Belgium

Human liver, modeling, vascular corrosion casting, micro-CT scanning, 3D reconstruction 


\section{Modelling of the hepatic circulation by combining vascular corrosion casting and micro-CT imaging}

Introduction: Hepatic perfusion plays a crucial role in many liver-related research areas (e.g. living donor liver transplantation, machine perfusion preservation, cirrhosis). Nevertheless, liver perfusion remains relatively poorly understood, especially at the microcirculation level. It is thus essential to clarify the hepatic vascular morphology and hemodynamics. Therefore, we visualised the liver macro- towards microvasculature and performed computer simulations of the hepatic circulation.

Methods and results: Vascular corrosion casting was applied to a human liver (discarded for transplantation) by simultaneous injections of resin (Batson' ${ }^{\mathrm{TM}}{ }^{\# 17}$, Polysciences, USA) in the hepatic artery and portal vein. Data on the liver macrovasculature were gathered by a high resolution (110 $\mu \mathrm{m})$ in globo micro-CT scan. Consecutive samples of different orders of magnitude were dissected from the cast and imaged at increasing resolutions, the most detailed scan (resolution $2.6 \mu \mathrm{m}$ ) obtained from a sample of $\pm 0.134 \mathrm{~mm}^{3}$. Image processing (Mimics, Materialise, Belgium) allowed segmentations and 3D reconstructions up to the sinusoidal network (Figure 1). These data were used to quantify branching topology and vessel features such as radii (up to 13 generations: range 13.2 to $0.08 \mathrm{~mm}$; sinusoids: 6.63 $\mu \mathrm{m}$ ) and lengths (range 74.4 to $0.74 \mathrm{~mm}$ ). Sinusoidal porosity was found to be $0.15 \pm 0.03$. Various computational models (electrical network analogues, detailed 3D computational fluid dynamic models) were used to model pressure drops and flows throughout the liver (Figure 2; results of electrical liver model for natural blood flow and hypothermic machine perfusion). Microcirculatory flow simulations revealed anisotropic permeability characteristics within liver lobules (higher permeability parallel to the central vein; lower permeability in radial or circumferential directions).

Conclusion: Combining vascular corrosion casting and micro-CT imaging allows (i) to quantify the hepatic vascular anatomy up to the microcirculation level, and (ii) to model hepatic perfusion. This approach may lead to novel insights into liver microcirculation, that can be used to study normal and pathological liver perfusion in the future.

Acknowledgment: This research was supported by the Agency for Innovation by Science and Technology in Flanders (IWT), Belgium.

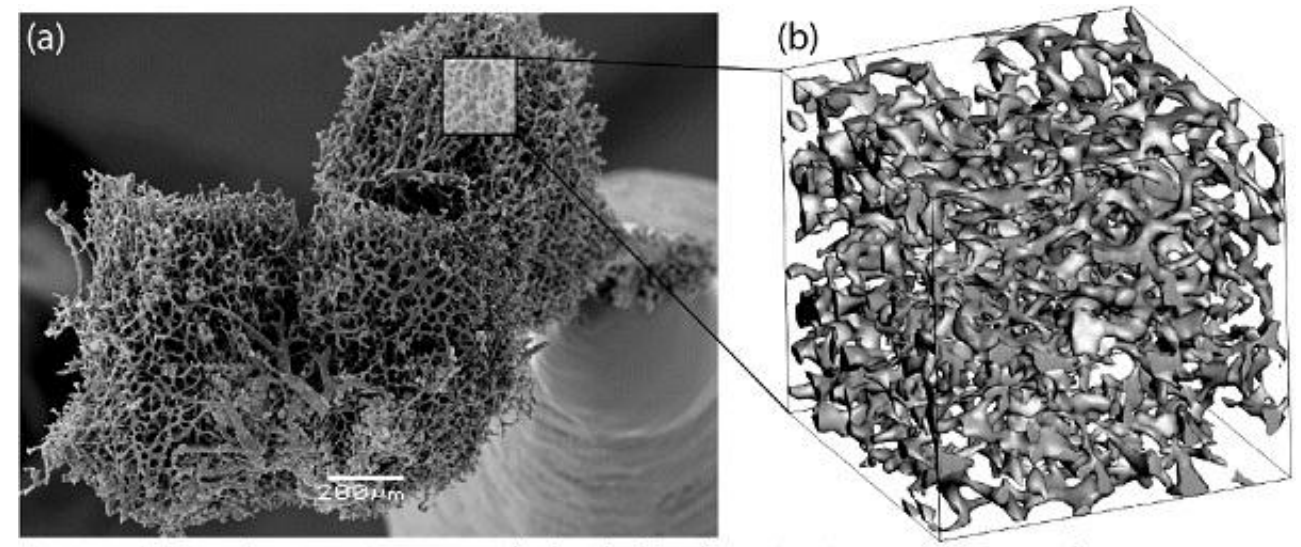

Figure 1. (a) Vascular corrosion cast at the level of the hepatic microvasculature and (b) $3 D$ reconstruction of a sinusoidal cube $\left(0.15 \times 0.15 \times 0.15 \mathrm{~mm}^{3}\right)$ 

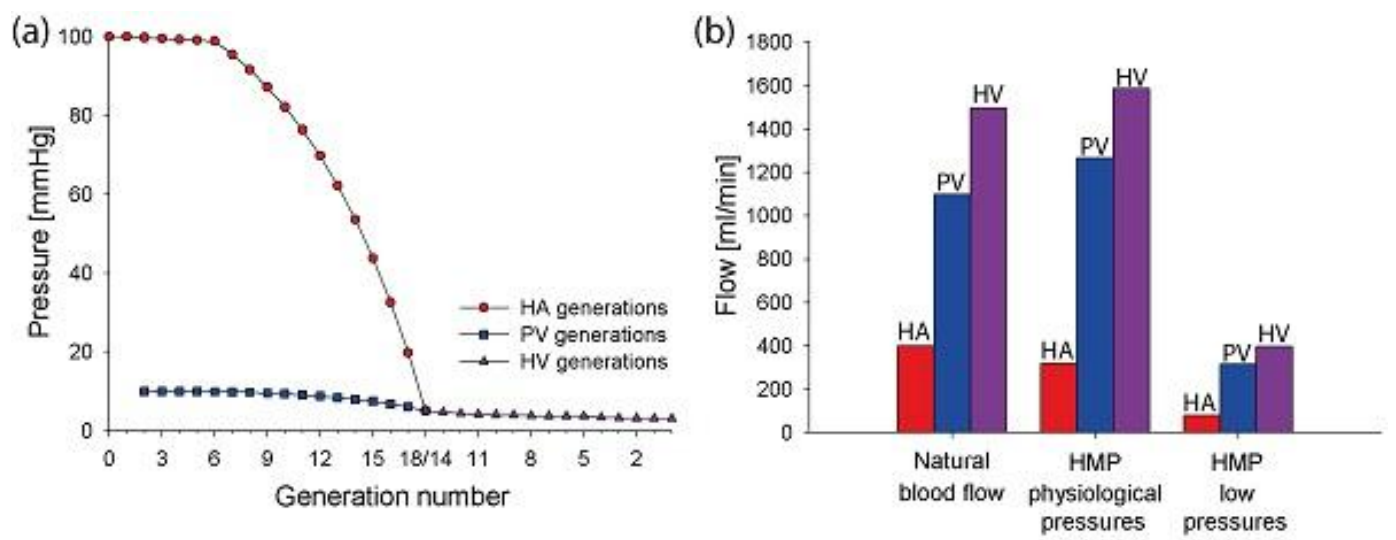

Figure 2. Results of the computational liver model: (a) Pressure profile throughout the liver for natural blood flow; (b) Hepatic arterial (HA), portal venous (PV) and hepatic venous (HV) blood flow in different conditions (natural blood flow, hypothermic machine perfusion (HMP)). 